\title{
Konzeption zur Vollständigkeitsprüfung von gefügten Strukturen am Beispiel Schienenfahrzeugwagenkästen
}

\author{
Uwe Jurdeczka \\ ALSTOM Transport Deutschland GmbH, Linke-Hofmann-Busch-Straße 1, 38239 Salzgitter, \\ Deutschland, uwe.jurdeczka@transport.alstom.com
}

\begin{abstract}
Zusammenfassung
Vollständigkeitsprüfung an Stahlbaustrukturen mit mehreren hundert Anbauteilen (wie zum Beispiel Wagenkästen/Karosserien für Schienenfahrzeuge) ist eine Herausforderung. Es gelingt, mit dem Einsatz von 3D Scannern ausreichend umfangreiche Information über die Ist-Konfiguration der zu prüfenden Stahlbaustruktur zu gewinnen. Mittels eines angepassten Algorithmus wird auf das jeweilige 3D Model als Soll Zustand mit Differenzbildern überlagert. Diese Differenzbilder zeigen eventuelle Abweichungen und Fehlteile an, mindestens jedenfalls die suspekten Stellen. Damit lassen sich die Nachteile der bisher praktizierten ausschließlich manuellen Vollständigkeitsprüfung, nämlich Ermüdung und Geschwindigkeit der Prüfer, weitgehend umgehen.
\end{abstract}

Keywords: Stahlbaustrukturen, Vollständigkeitsprüfung, 3D Laserscanner, Auswertealgorithmus, Differenzbilder

\section{Motivation}

Die Vollständigkeitsprüfung an Stahlbaustrukturen mit mehreren hundert Anbauteilen (wie zum Beispiel Wagenkästen/Karosserien für Schienenfahrzeuge) ist eine Herausforderung. Die Taktzeit und die konstruktive Variantenvielfalt mit geringer Stückzahl völlig baugleicher Wagenkästen sind die motivierenden Randbedingungen. Mittels MES - Manufacturing Execution Systems beherrscht man diese Variantenvielfalt in der Fertigung. Die entsprechende fertigungsbegleitende Prüfung erfordert ebenso Aufmerksamkeit. Die Variantenvielfalt besteht in der Art der Anbauteile, ihrer Anzahl und in ihrer Positionierung. Circa dreihundert Anbauteile (Halter, Laschen, Erdungsanschlüsse u.ä.) sind innerhalb der Taktzeit von einer Schicht (ca. 8h) auf Vollständigkeit zu prüfen. Zwar werden fertigungsbegleitende Vollständigkeitsprüfungen seit langem ausgeführt und dem Prüfpersonal wurden viele Hilfsmittel bereitgestellt (z.B. Checklisten, Sektoreneinteilung der Prüfobjekte bis hin zu Prüfzeit/PausenzeitRegimes). Doch im Wesentlichen ist diese Vollständigkeitsprüfung bis heute eine manuelle Sichtprüfung. Gelegentlicher Prüfschlupf führt zu Mehraufwand in den folgenden Arbeitsgängen. Mit der Entwicklung von Sensoren und von Bildverarbeitungssystemen wurden weitere Hilfsmittel möglich, um das Prüfpersonal zu entlasten und um die Fehlerquote der manuellen Sichtprüfung zu verbessern.
Die Entwicklung von gerätegestützten Vollständigkeitsprüfungen schreitet voran. Erfahrungsberichte für erste Entwicklungen hin zu einer gerätegestützten und modellbasierten Vollständigkeitsprüfung, für kleinere Prüfvolumen als die hier vorgestellten, liegen vor [1]-[4]. Die bekannten Ansätze sind also weiterzuführen, um ein Prüfverfahren zu beschreiben, das mit einem angemessenen Geräteeinsatz (Investition eines Mannjahres) auskommt, und welches die gegebene Taktzeit (eine Schicht, acht Stunden) nicht stört. Modellbasierte Prüfungen versprechen nun die Möglichkeit, auf die Variantenvielfalt der Konstruktion und Fertigung mit einfach anzupassenden Prüfplänen und Merkmalslisten einzugehen.

Bereits beschrieben ist das Generieren von Differenzbildern aus dem Vergleich 3D CAD Daten zu 3D Ist Daten. Dabei geht man bisher nicht auf die der Fertigung zugestandenen Toleranzen und deren Aus-nutzung ein. So führt beispielsweise eine Längenabweichung der realen Stahlbaukonstruktion versus Datensatz zu einer Anzeige im Differenzbild. Allerdings wäre eine solche Abweichung nicht unbedingt ein Fehler, sondern eine als noch i.O. bewertete Fertigungsstreuung. So könnte eine ganze Seite einer kubischen Stahlbaukonstruktion (z.B. Seitenwand eines Schienenfahrzeuges) als fehlend/abweiาchend dargestellt werden. Dies, obwohl kein Fehler vor- 
liegt, sondern die Toleranz auf das Nennmaß ausgenutzt wird.

Die vorhandenen Geräteklassen und Auswerteverfahren sind also zu modifizieren und zu adaptieren bezüglich:

- der Erfassung des Ist-Zustandes des Prüfobjektes (z.B. Punktwolke, 3D Scan), und

- des Auswertealgorithmus.

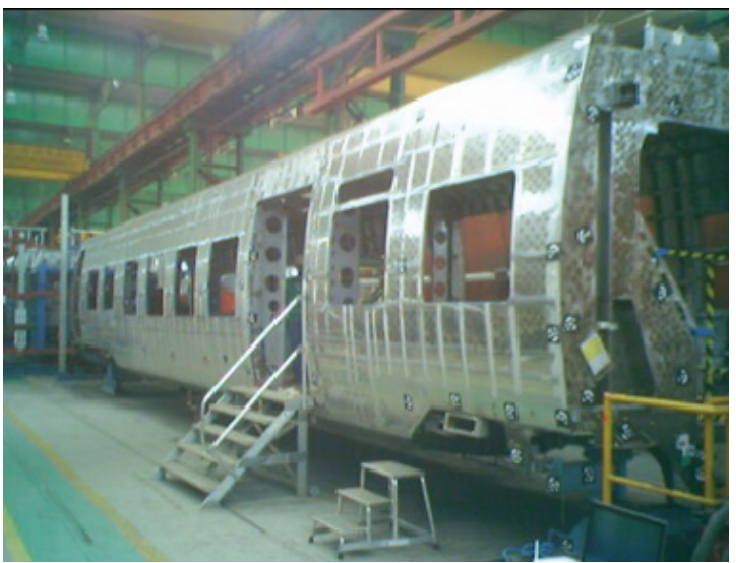

Abb. 1: Ein Wagenkasten als Beispiel für Prüfobjekt im Prüfstand

\section{Lösungsansatz}

Das hier präsentierte Konzept stellt ein Prüfsystem für die Vollständigkeitsprüfung von Anbauteilen vor. Es gelingt, eine Aussage über den Ist-Zustand des Prüfobjektes zu erhalten, ganz besonders über die Vollständigkeit der Anbauteile.

Optische Verfahren scheinen wegen der Ausdehnungen der Prüfobjekte gut geeignet, da damit in kurzer Zeit eine große Anzahl von Informationen über das Prüfobjekt gewonnen werden kann. Auf Abschattung von Merkmalen (Hinterschneidungen) kann man durch MultiSensor-Systeme oder wie hier praktiziert, durch Bewegung des Sensors innerhalb des Prüfobjektes eingehen.

\section{Erfassung des Ist-Zustandes}

Im Gegensatz zu den bereits beschriebenen Verfahren wird hier ein terrestrischer 3D Laser Scanner (TLS, auch HDS - High Definition Surveying genannt) zur Datenakquise (3D Scan, Punktwolke) genutzt. Schnelligkeit, vergleichsweise einfache Bedienung und handhabbare Punktwolken sind die Vorteile aus der Verwendung eines TLS/HDS zur Gewinnung des Abbildes des Prüfobjektes.
Sehr wichtig ist wegen der Größe des Prüfobjektes: es kann für die hier angestrebten Genauigkeiten auf Probenvorbereitung (Reinigung, Mattierung u.ä.) verzichtet werden.

Dabei werden die Geräteparameter an das Prüfobjekt soweit angepasst, dass die Qualität der gescannten Punktwolke (Punktdichte, Intensität, enthaltene und weitgehend automatisch erkannte Targets) für die weitere Auswertung mindestens ausreichend ist.

Die Filtereinstellung für sogenannte Mixed Points ist ebenfalls von Bedeutung. Wenn der Laserstrahl des TLS/HDS auf eine Kante trifft und sowohl von dieser Kante als auch von der eventuell dahinter liegenden Fläche reflektiert wird, nennt man dies Mixed Point. Bei kurzen Abständen von Kante zur dahinterliegenden Fläche wird der Mixed Point nicht immer zuverlässig erkannt und von der weiteren Auswertung ausgeschlossen.

Die teilweise stark reflektierenden kaltgewalzten Stahloberflächen stellen hohe Anforderungen an die Parameterwahl für Intensität und Auswertefilter. Säulen im Inneren führen zu Abschattungen. Ein typischer Wagenkasten eines Regionalzuges kann mit fünf bis sechs verschiedenen Sensorpositionen im Innenraum gescannt werden, um die Abschattungen zu umgehen.

\section{Tab. 1: Zeitbedarf für Scannen und Prüfen (Scannen und Rüsten) bei einer Erfassung mit Punkt $\neg$ dichte von 6mm@10m für verschiedene Anzahl von Sensorpositionen.}

\begin{tabular}{|c|c|c|}
\hline $\begin{array}{c}\text { Anzahl } \\
\text { der } \\
\begin{array}{c}\text { Sensor- } \\
\text { positionen }\end{array}\end{array}$ & $\begin{array}{c}\text { Dauer des } \\
\text { Scannens bei } \\
\text { Punkt } \text {-dich- } \\
\text { ten von } \\
6 m m @ 10 m\end{array}$ & $\begin{array}{c}\text { Gesamt- } \\
\text { prüfzeit } \\
\text { einschließlich } \\
\text { Rüsten }\end{array}$ \\
\hline 5 & 10 Minuten & 30 Minuten \\
\hline 6 & 12 Minuten & 35 Minuten \\
\hline
\end{tabular}

Verglichen mit den bisher praktizierten bis zu 8h Vollständigkeitsprüfung ergibt sich hier ein großes Potential an Zeiteinsparung, auch wenn man bedenkt, dass die Auswertung der gescannten Punktwolke nicht vollständig während der Erfassung ablaufen kann, sondern bis zu zwei Stunden nachläuft.

Die Anbauteile sind unterschiedlich groß. Die kleineren haben eine Ausdehnung von typischerweise $15 \mathrm{~mm} \times 25 \mathrm{~mm} \times 1 \mathrm{~mm}$. Diese Laschen sollten also mit mehreren Antastungen des 3D Scanners belegt werden, um für den anschließenden Auswertealgorithmus aus- 
reichend Ist-Daten zur Verfügung zu haben. An den 3D Scannern einstellbare Erfassungsdichten von 6mm@10m führen bei ca. 2m Scanabstand zu Punktdichten in der Erfassung von ca. $1,2 \mathrm{~mm}$. Dies erwies sich für das Wagenkasteninnere als ausreichend.

Es finden zwei verschiedene Prinzipien bei der Entfernungsmessung Anwendung.

a) Impulslaufzeit

Es wird kurzzeitig ein Lichtimpuls vom Laserscanner ausgesandt und nach dem Durchlaufen der Strecke zum Objekt und wieder zurück mit einer Zeitverzögerung, die proportional zur Streckenlänge ist, wieder vom Laserscanner empfangen.

b) Phasenlaufzeit

Beim Phasenlaufzeitverfahren wird kontinuierlich ein moduliertes Laserlicht ausgesandt und permanent die Phasenlage der Modulationswelle des reflektierten Lichtes bestimmt. Die Phasenlage ist proportional zur Länge eines Reststückes der gesamten Objektentfernung. Dieses Reststück kann maximal soll lang sein wie die Wellenlänge der Modulationswelle. Von der Gesamtentfernung bleibt so regelmäßig ein Anteil unbestimmt, der einem ganzzahligen Vielfachen der Modulationswellenlänge entspricht. Dieses so genannte Mehrdeutigkeitsproblem wird durch die Verwendung verschiedener Modulationswellen behoben [5].

Wie die Geräte-Parameter ans Prüfobjekt (glänzende Oberflächen, für die Geräteklasse vergleichsweise nah liegender Fokusbereich) angepasst werden können, wurde in Versuchen ermittelt. Dabei sind verschiedene Wellenlängen des abtastenden Laserstrahls, unterschiedliche Strahldurchmesser und auch geräteinterne Auswerteverfahren des reflektierten Strahls zu berücksichtigen.

Für die Verfahrensuntersuchung stellte Leica Geosystems mit der ScanStation P40 einen Laufzeitscanner neuester Bauart zur Verfügung (Leica Geosystems $\mathrm{GmbH}$ Vertrieb, Düsseldorf). So konnte in einer Versuchsreihe die Eignung für die Oberflächen und Geometrien bestätigt werden.

In Abb. 2 ist die damit gewonnene Punktwolke aus einer von fünf Sensorpositionen zu sehen.

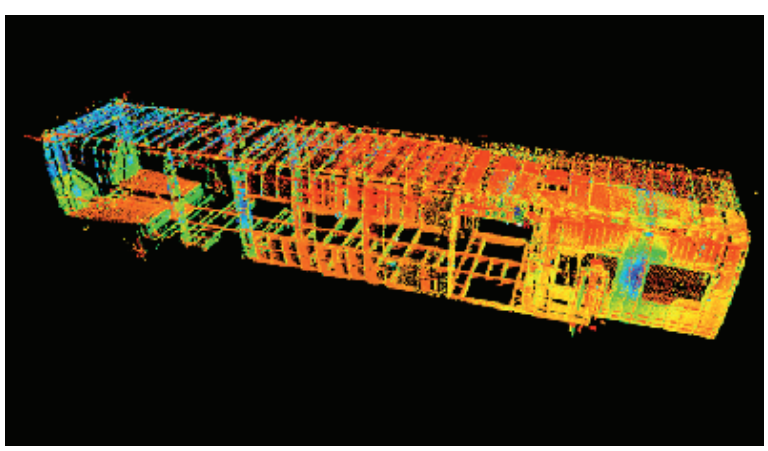

Abb. 2: Beispiel Punktwolke. Dargestellt ist die Punktwolke im Ergebnis des Scannens von einer Sensorposition(von fünf Positionen insgesamt für diesen Wagenkastentyp), die Farbmarkierung drückt die Intensität der Reflektion (Remission) aus.

Diese Eignung wurde anhand des von der LIMESS Messtechnik und Software GmbH bereitgestellten Surphaser 25HSX-IR (LIMESS Messtechnik \& Software GmbH, Krefeld) auch für Phasenscanner in einer weiteren Versuchsreihe nachgewiesen.

Verschiedene Scanner-Bauarten lassen sich also für die Prüfaufgabe einsetzen, entsprechendes Setup und auf den Messbereich angepasste Konfiguration vorausgesetzt.

Da aus verschiedenen Sensor-Positionen aufgenommen wird, sind zunächst die Einzelscans zu einer gemeinsamen Punktwolke zusammen zu fassen. Die so mit den Scannern gewonnene Punktwolke ist eine detailreiche Abbildung des tatsächlich gebauten Zustandes (Ist-Zustand).

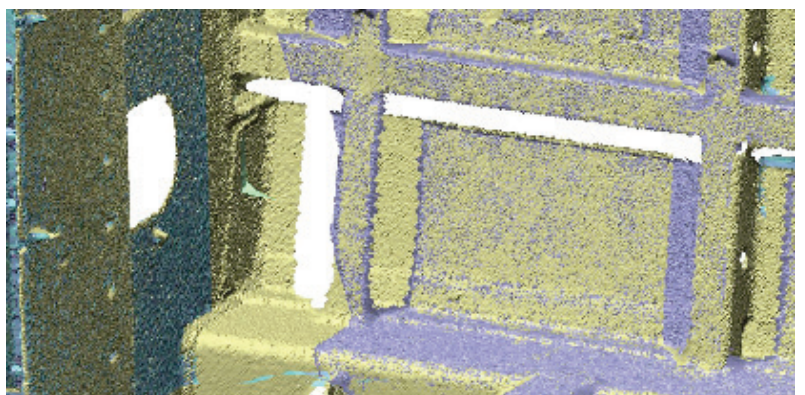

Abb. 3: Resultat des Best-Fit zur Reduzierung der Klaffungen der Scans aus den verschiedenen Sensor-Positionen (Scan eins: gelb, Scan zwei: violett) (Software PolyWorks). 
Der Soll-Zustand wird aus den vorhandenen 3D-Konstruktionsmodellen eingespielt.

Vergleiche zwischen der gescannten Punktwolke und dem 3D Modell führen zur Generierung von Differenzbildern.

Diese Differenzbilder stellen Informationen über die Vollständigkeit bereit.

Die Generierung der Differenzbilder erfolgt nach einem neu entwickelten Algorithmus. Dieser Algorithmus verbindet den Funktionsumfang von entsprechenden Programmpaketen und erweitert ihn.

Programmpakete für Punktwolkenbetrachtung, -veränderung und auch für die Erzeugung von Differenzbildern bestehen bereits. Neu ist zunächst die bereichsweise Betrachtung sowohl des 3D Modells als auch der korrespondierender Punkte in der Punktwolke.

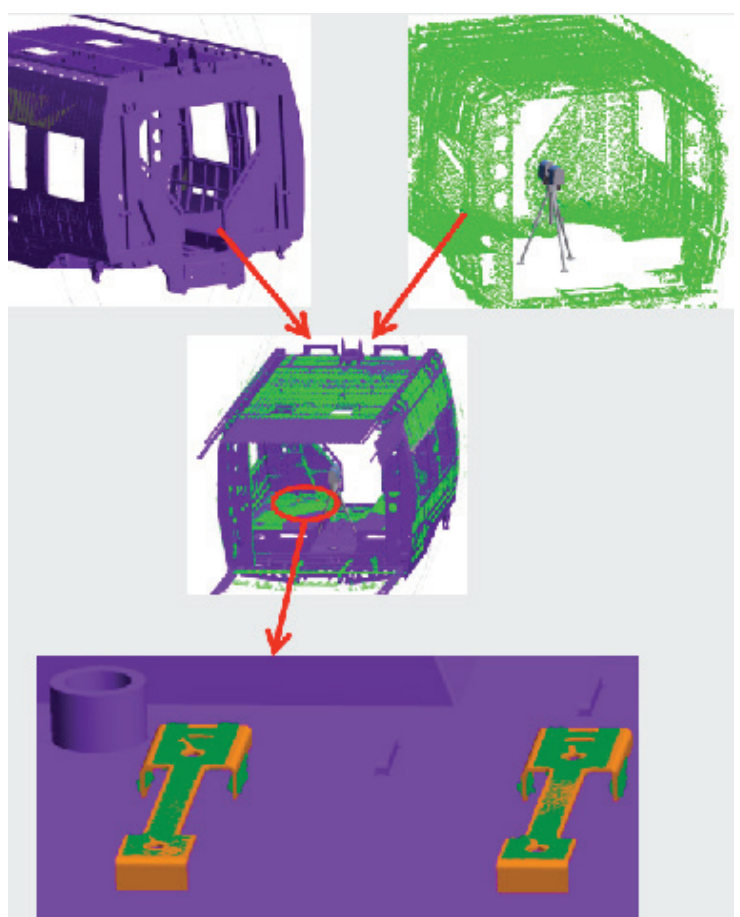

Abb. 4: Beispiel für zwei Anbauteile, Merkmal i.O. laut Vergleich CAD gegen Punktwolke. (Software SpatialAnalyzer).

\section{Betrachtung zum Toleranzfeld der Grundstruktur, die die Anbauteile trägt}

Ein Prüfsystem sollte keine Fehlanzeigen verursachen. Dazu gilt es, die Toleranzfelder, sowohl für Positionierungen der Anbauteile, als auch für die Positionierung der Strukturelemente, auf denen sich die Anbauteile befinden, zu beachten. Das Beachten dieser möglichen und akzeptierten Fertigungstoleranzen zum Nennmass vermeidet die Ausgabe von Fehleranzeigen zweiter Art. Denn immerhin ist das
Toleranzfeld der Wagenkastenlänge bei einem Nennmass von $18 \mathrm{~m}$ nach Tabelle1, Zeile B in DIN EN ISO $13920+/-14 \mathrm{~mm}$ breit. Und damit größer als die Ausdehnung der an der Rückwand angebrachten Anbauteile. So kann also nicht die komplette gescannte Punktwolke mit dem kompletten 3D Modell verglichen werden. Vielmehr muss der Vergleich bereichswiese erfolgen.

\section{Zum Algorithmus}

Die Punktwolke aus den 3D Scans ist zunächst aufzubereiten, nämlich Registrierung (Zusammenführen) der Einzelscans aus den verschiedenen Sensorpositionen und Vermaschung. Beispielparameter für die Vermaschung: Netzgröße maximal $10 \mathrm{~mm}$ und Abweichung Einzelpunkt $0,1 \mathrm{~mm}$. Optional kann auch eine Beurteilung der Punkte nach Scanner Abstand und besonders nach Auftreffwinkel erfolgen.

Innerhalb des zu untersuchenden Bereichs wird die Punktwolke im Best Fit auf den Datensatz gelegt.

i.) Es wird ein Abstand $\varepsilon$ definiert, innerhalb dessen Punktwolke und 3D Modell als in Relation stehend betrachtet werden.

ii.) Nun werden alle Elemente des 3D Modells geprüft, ob es korrespondierende Punkte (Abstand kleiner $\varepsilon$ ) in der Punktwolke gibt. Dabei wird nicht nur ein Abstand als Suchkriterium verwendet, sondern auch eine Winkeluntersuchung durchgeführt. Optional kann eingestellt werden, dass gegen das höchste CADElement verglichen wird, d.h. es werden keine innenliegenden oder entgegen der Scanpunkte orientierten CAD-Elemente verwendet. Dies minimiert die Fehleranfälligkeit besonders bei komplexen CADAssemblies.

iii.) Die CAD-Elemente mit korrespondierenden Punkten in der Punktwolke werden markiert.

iv.) Die markierten Elemente sollen vorhanden sein, und sind vorhanden.

v.) Die Auswahl der Elemente des 3D Modells wird im nächsten Schritt umgekehrt/invertiert. Jetzt sind alle 3D Modell Elemente markiert, die keine Entsprechung in der Punktwolke haben.

vi.) Diese Elemente sollen vorhanden sein, sind aber laut Punktwolke nicht vorhanden.

vii.) Die so gewonnene Auswahl von 3D Modell Elementen zeigt also genau jene an, die eventuell fehlen, falsch positioniert sind, also mindestens suspekt sind und einer weiteren Betrachtung bedürfen. 
Die Hypothese zu diesen nachzuprüfenden Merkmalen ist: Nicht vorhanden, bzw. außerhalb der tolerierten Fehlerschranke positioniert.

Eine manuelle Nachprüfung ist dann einfach und ausreichend schnell möglich, soweit die Anzahl der Prüfmerkmale von mehreren hundert auf deutlich weniger als einhundert reduziert wurde, nämlich durch die Anwendung des hier beschriebenen Workflows. Dazu zeigt Abb. 5 eine Auswertung Seitenwand.

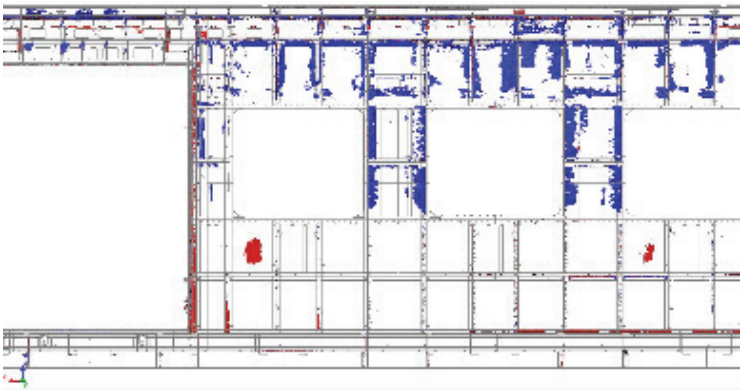

Abb. 5: Ausschnitt Seitenwand innen. Dargestellt ist der 3D Datensatz, die Farbmarkierung codiert den Abstand der korrespondierenden Punkte der Punktwolke (Software PolyWorks).

An vergleichsweise vielen Stellen in Abb. 5 ist dieser Abstand größer als $\varepsilon$ und wird hier also in der Farbmarkierung sichtbar. Eine Manuelle Prüfung der suspekten Stellen in der Seitenwand ist nötig. Hier macht sich also das Rauschen in der Erfassung der Punktwolke bemerkbar und kann durch den Algorithmus nur teilweise ausgeglichen werden.

Der für das jeweilige Prüfobjekt zu definierende Abstand $\varepsilon$ und die Streuung der erfassten Punkte um den jeweils wahren Positionswert definieren also gemeinsam die Qualität der Anzeige im Differenzbild.

Die Anwendung des Algorithmus auf das Dach in Abb. 6 zeigt ein deutlich schärferes Ergebnis. Unterschiedliche Aufnahmeparameter sind dafür teilweise verantwortlich. Nur an wenigen Stellen ist der Abstand Punktwolke zu 3D Datensatz größer als $\varepsilon$. Entsprechend werden wenige kleine Stellen in der Farbmarkierung sichtbar. Bereits in der Auswertung des Differenzbildes lässt sich die Aussage ableiten: Kein An bau $\neg$ teil ist so dicht mit Fehlpunkten belegt, dass von seinem Fehlen ausgegangen werden müsste. Diese Aussage aus dem gerätegestützen Prüfsystem unterstützt die Arbeit des Prüfers bei der Vollständigkeitsprüfung sehr.

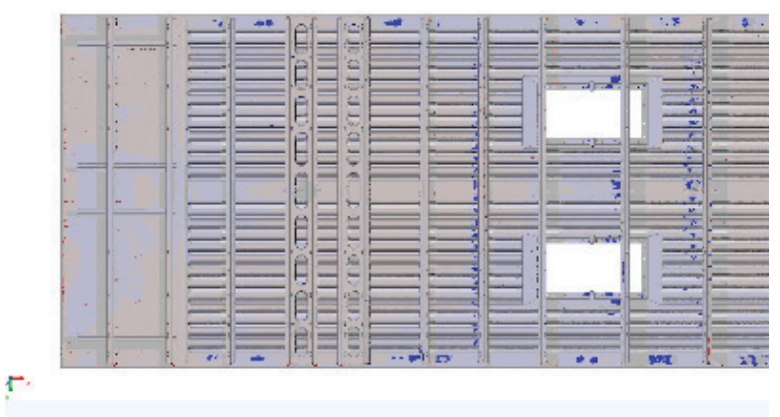

Abb. 6: Ausschnitt Dachsegment innen. Dargestellt ist der 3D Datensatz, die Farbmarkierung codiert den Abstand der korrespondierenden Punkte der Punktwolke (Software PolyWorks).

Die Ausgabe von Differenzbildern ist also wesentlich. Wichtig für die Lösung der hier besprochenen Prüfaufgabe ist zudem die Möglichkeit, neben Best Fit eine iterative Ausrichtung der Punktwolke gegen CAD mittels Relationships vorzunehmen (Ausgleichung mit Nebenbedingungen von definierten Beziehungen, z.B. RPS oder Einschränkungen nur in bestimmten Achsrichtungen ). Dies ermöglicht den Umgang mit dem grundsätzlichen Problem, dass die reale Länge des Wagenkastens mit dem Nennmaß aus dem CADDatensatz nur innerhalb der Toleranzen von mehreren Millimetern übereinstimmt. Eine Lösung ist: einzelne Sektionen der Punktwolke iterativ gegen CAD fitten und anschließend jedes Punktwolken-Segment gegen CAD vergleichen. Die Vollständigkeitskontrolle lässt sich nicht nur visuell sondern auch statistisch dokumentieren. Soweit Scripte erzeugt werden können, sind die Prozesse in der Software auch zu automatisieren.

Ob der Algorithmus sich in verbreiteten Software Programm Paketen darstellen lässt, wurde ebenfalls untersucht. Aus Voruntersuchungen ist bekannt, dass die Möglichkeit des Handlings von sehr großen Punktewolken zur Ausgabe von Differenzbildern doch variiert.

Mit Polyworks, bereitgestellt von Fa. Duwe-3d $A G$, Lindau, mit SpatialAnalyzer, bereitgestellt von Fa. VMT GmbH, Bruchsal, und mit 3D Reshaper, bereitgestellt von Fa. Technodigit wurden sehr zufriedenstellende Ergebnisse erzielt. Diese Aussagen über die Software Programm Pakete wurden in jeweils einer Testphase gewonnen. 


\section{Erfahrungen und Ausblick}

Mit der Ausgabe von Differenzbildern und den darin markierten Fehlteilen gelingt also eine von Ermüdung und Erfahrung des Prüfers weitgehend unabhängige Vollständigkeitsprüfung.

Von den ca. dreihundert Anbauteilen werden viele als richtig und vorhanden erkannt.

Detektierte Fehler werden innerhalb des Modells nachvollziehbar und gut auffindbar dargestellt.

Eine manuelle Nachkontrolle an diesen vergleichsweise wenigen Stellen separiert dann echte Fehleranzeigen von den gelegentlichen Fehlern höherer Ordnung.

Da die Sollbeschreibung mittels 3D CAD Daten Modell erfolgt, kann die Prüfmethode auch als modellbasiert eingeordnet werden.

Die modellbasierte Sollbeschreibung führt zu hoher Flexibilität bei Änderung an den Prüfobjekten (Wagenkästen) und somit zu einer besonderen Eignung für variantenreiche Montageprozesse. Konstruktive Änderungen im CAD Modell können so auch kurzfristig in den Prüfplan (Liste der zu prüfenden Merkmale) einfließen. Besonders wichtig für den Umfang der Rechenoperationen ist die Struktur der 3DModell Daten. Soweit es gelingt, durch sinnvolle Strukturierung jeweils ein sogenanntes Light Model mit den Anbauteilen separat vom kompletten Konstruktionsdatensatz auszugeben, stehen geeignete Eingangsinformationen über den Soll Zustand zur Verfügung. Das Light Model lässt sich als Auszug aus der Stückliste verstehen, das also alle Anbauteile aufführt. Der Im- und Export von Prüfmerkmalsplänen aus dem Light Model kann dann gut unterstützt werden. So können also Prüfmerkmalspläne weitgehend automatisiert entstehen (Anbauteil-Name, Identnummer, weiteres).

Die bisher bekannten Einsatzgrenzen von Prüftechnik (Datenakquise) und Grenzen aus der softwareseitigen Weiterverarbeitung der Informationen (also dem post processing) wurden erweitert, sodass nun in der Fertigungspraxis eine weitere Prüfaufgabe gerätegestützt durchgeführt werden kann.

Bestandteil des Prüfablaufes ist eine Einteilung des Prüfobjektes in Bereiche und zwar sowohl im Datensatz als auch in der Punktewolke. Für diese geometrischen Bereiche können für minimales Rauschen optimale geometrische Bereichsgrößen ermittelt werden. Hier sind weitere Untersuchungen nötig.
Das Rauschverhalten kann durch Einstellung so beeinflusst werden, dass die sichere Detektion von Merkmalen von typischerweise $15 \mathrm{~mm}$ $x 25 \mathrm{~mm} \times 1 \mathrm{~mm}$ gelingt. Die Erzeugung von Differenzbildern nach dem vorgestellten Algorithmus weist die Fehler (fehlende Anbauteile, deutliche Fehlpositionierung) sehr zuverlässig nach. Siehe dazu den Vergleich von Abb. 5 zu Abb. 6.

Das Prüfsystem wurde bereits versuchsweise innerhalb einer taktenden Fertigungslinie verwendet.

Die Ergebnisse sind vielversprechend. Daraufhin wurde ein Innovationsprojekt innerhalb Alstom gestartet und es wurde die Zusammenarbeit mit dem Institut für Produktionsmesstechnik der TU Braunschweig vereinbart.

Den hier und oben im Text genannten Instituten/Firmen ist für die Unterstützung der bisherigen Entwicklung sehr zu danken.

\section{Literaturnachweis}

[1] Dirk Berndt, Ralf Warnemünde: Flexible modellbasierte Montageprüfung, Prüfung durch Vergleich mit synthetischen Messdaten. http://www.iff.fraunhofer.de/de/geschaeftsbereic he/messtechnik-prueftechnik/montagepruefungmodellbasiert.html; 2012;

[2] Kelch, Johannes: Vollautomatisierte 100\% Inspektion von Karosserieteilen-Aus allen Perspektiven. Carl-Hanser Verlag München, QZ Jahrgang 60 (2015) 05; Seite 62; www.qzonline.de/974021

[3] Bevor Fehler entstehen, 3D Bildverarbeitungssystem prüft Schaumstoffteile. Carl-Hanser Verlag München, QZ Jahrgang 60 (2015) 11; Seite 64; auch www.qzonline.de/1047972

[4] Cem Tarih: Aktueller Stand der Automatisierung von Sichtprüfungen, speziell Vollständigkeitsprüfungen an großen Stahlbaustrukturen am Beispiel Schienenfahrzeugwagenkästen. Studienarbeit an der Ostfalia Hochschule für angewandte Wisschenschaften, Hochschule Braunschweig/ Wolfenbüttel, Fakultät Maschinenbau; 2016

[5] Fredie Kern: Terrestrisches Laser Scanning. 2007; http://www.architekturvermessung.de/05Literatur/doc/laserscanning_fkern.pdf 